\title{
Étude comparative des peuplements ichtyologiques de l'Aire Marine Protégée de Joal-Fadiouth et des pêcheries des zones du pourtour non protégées à l'exploitation halieutique
}

\section{Serigne Modou Sarr, PhD}

Maître de Conférence-CAMES, Département Productions Forestières, Institut Supérieur de Formation Agricole et Rurale (ISFAR),

Université Alioune Diop. Bambey, Sénégal

\section{Mamadou Ndiaye, Doctorant}

Conservateur des Parcs Nationaux, Direction des Aires Marines Protégées, Ministère de l'Environnement et du Développement Durable, Sénégal

\section{Alassane Thiam, PhD}

Maître-Assistant, Unité de Formation et de Recherche (UFR) Sciences de l'Ingénieur, Université Pr. Iba Der THIAM de Thiès, Sénégal

\section{Fokina Mane}

Ingénieur des travaux des eaux et forêt, Direction des Eaux et Forêts, Ministère de l'Environnement et du Développement Durable, Sénégal

\section{Doi:10.19044/esj.2021.v17n17p133}

Submitted: 31 March 2021

Accepted: 04 May 2021

Published: 31 May 2021
Copyright 2021 Author(s)

Under Creative Commons BY-NC-ND

4.0 OPEN ACCESS

Cite As:

Sarr S.R., Ndiaye M., Thiam A. \& Mane F. (2021). Étude comparative des peuplements ichtyologiques de l'Aire Marine Protégée de Joal-Fadiouth et des pêcheries des zones du pourtour non protégées à l'exploitation halieutique. European Scientific Journal, ESJ, 17(17), 133. https://doi.org/10.19044/esj.2021.v17n17p133

\section{Résumé}

L'effondrement des stocks de poisson est dû à la surexploitation des peuplements ichtyologiques consécutifs aux mauvaises pratiques de pêche au Sénégal. Cette situation a amené les écologistes marins et les gestionnaires de l'environnement à réévaluer les méthodes traditionnelles de gestion des ressources halieutiques. Pour connaitre la dynamique des peuplements ichtyologiques, nous avons mené une étude comparative des peuplements ichtyologiques de l'Aire Marine Protégée de Jaol-Fadiouth et des pêcheries des zones non protégées à l'exploitation halieutique durant la saison froide 2015. Ainsi, l'exploitation des données de pêches expérimentales réalisées au 
niveau de 16 stations dans les deux zones a permis de faire l'étude. L'analyse des paramètres environnementaux relevés sur chaque station montre que la zone de l'aire marine protégée est similaire à celle des zones non protégées à l'exploitation halieutique. Par conséquent, les éventuelles différences de peuplement ne s'expliquent pas par la variabilité des paramètres physicochimiques. L'analyse de paramètres physico-chimiques, des indicateurs de biodiversité à savoir : la richesse spécifique, les indices de diversité de Shannon-Weaver et d'équitabilité Pielou ont permis de déterminer les caractéristiques environnementales, la composition et la nature des peuplements, d'évaluer l'impact des mesures de gestion des différentes zones.

Mots clés : Diversité, aire marine protégée, pêcheries, gestion

\title{
Comparative Study of the Ichthyological Populations of the Jaol-Fadiouth Marine Protected Area and the Fisheries of Areas not Protected from Fishing Exploitation
}

\author{
Serigne Modou Sarr, PhD \\ Maître de Conférence-CAMES, Département Productions Forestières, \\ Institut Supérieur de Formation Agricole et Rurale (ISFAR), \\ Université Alioune Diop. Bambey, Sénégal \\ Mamadou Ndiaye, Doctorant \\ Conservateur des Parcs Nationaux, Direction des Aires Marines Protégées, \\ Ministère de l'Environnement et du Développement Durable, Sénégal \\ Alassane Thiam, PhD \\ Maître-Assistant, Unité de Formation et de Recherche (UFR) Sciences de \\ l’Ingénieur, Université Pr. Iba Der THIAM de Thiès, Sénégal

\section{Fokina Mane}

Ingénieur des travaux des eaux et forêt, Direction des Eaux et Forêts, Ministère de l'Environnement et du Développement Durable, Sénégal

\begin{abstract}
The collapse of fish stocks is due to the overexploitation of fish populations as a result of poor fishing practices in Senegal. This situation has led marine ecologists and environmental managers to re-evaluate traditional methods of managing fishery resources. In order to know the dynamics of the ichthyological populations, we conducted a comparative study of the ichthyological populations of the Jaol-Fadiouth Marine Protected Area and the fisheries of the areas not protected from fishing during the 2015 cold season. Thus, the exploitation of data from experimental fisheries carried out at 16
\end{abstract}


stations in the two areas allowed the study to be carried out. The analysis of the environmental parameters recorded at each station shows that the area of the marine protected area is similar to that of the areas not protected from fisheries exploitation. Therefore, any differences in population size cannot be explained by the variability of physico-chemical parameters. The analysis of physico-chemical parameters, biodiversity indicators such as species richness, the Shannon-Weaver diversity index and the Pielou equitability index allowed us to determine the environmental characteristics, the composition and the nature of the populations, and to evaluate the impact of the management measures of the different areas.

Keywords: Diversity, marine, protected, fisheries, management

\section{Introduction}

Au Sénégal, le secteur de la pêche représente 1,8\% du Produit Intérieur Brut (PIB) (ANDS, 2015). En outre, elle emploie près de $20 \%$ de la population active, soit environ 600000 personnes, et apporte plus de $75 \%$ des protéines animales consommées $(B a, 2000)$. A la lumière de ces études, il apparaît qu'un effondrement des stocks halieutiques aurait des conséquences sociales et économiques dramatiques pour le Sénégal. Or jusqu'à présent, les tentatives de l'administration des pêches et de leurs partenaires n'ont pas permis d'inverser la tendance à la diminution des ressources (ANDS, 2011). L'évaluation de la dynamique des peuplements ichtyologiques devient un défi important de nos jours au regard des problèmes liés aux changements climatiques, à la forte pression de l'exploitation ressources itchyofauniques, à la destruction des habitats par les mauvaises pratiques de pêche (Sarr, Thiaw et Mbengue , 2018). Ainsi les résultats du diagnostic de l'état des stocks ciblés sur le littoral sénégalais effectué en 2005 par la FAO-COPACE (Organisation des Nations Unies pour l'alimentation et l'agriculture-Comité des pêches pour l'Atlantique Centre-Est) et ceux issus des travaux de l'ANSD (Agence Nationale de la Statistique et de la Démographie) en 2011 ont mis en évidence les caractéristiques de la crise des pêcheries sénégalaises. A cet effet, la protection et l'amélioration de la productivité des composantes ressources et habitats se posent avec beaucoup d'acuité (Hansen, 2010 ; Pauly et Froese, 2012). C'est pour répondre à cette situation et soucieux de la préservation de sa biodiversité que le gouvernement du Sénégal a manifesté la volonté politique de mettre en œuvre les recommandations du Congrès sur les parcs nationaux par la création d'aires marines protégées dont celle de Joal-Fadiouth ) par décret 2004-1408 du 04 novembre 2004. Ce nouvel outil d'aménagement des pêcheries vient ainsi renforcer les dispositions de gestion des ressources marines et côtières qui remontent vers les années 1960. Cependant, depuis sa création, l'AMP de Joal- Fadiouth n'a pas eu à faire une étude comparative 
des peuplements ichtyologiques avec ceux des pêcheries des zones non protégées à l'exploitation halieutique sur une saison hydro-climatique. C'est dans ce contexte que nous avons mené des travaux de recherches sur «étude comparative des peuplements ichtyologiques de l'Aire Marine Protégée de Jaol-Fadiouth et des pêcheries des zones non protégées à l'exploitation halieutique durant la saison froide $2015 »$. Ce travail a permis d'une part à mesurer les effets potentiels et l'efficacité de la gestion des ressources ichtyologiques de l'AMP comparée aux zones non protégées à l'exploitation halieutique. Il constitue un outil de gestion et d'informations pour l'état du Sénégal. L'objectif général de la recherche est d'évaluer l'efficacité de la gestion de l'AMP par une approche biologique (diversité spécifique de peuplement ichtyologique) comparées avec les pêcheries des zones non protégées à l'exploitation halieutique durant la saison hydro-climatique froide de l'année 2015. Les travaux ont permis de comparer plusieurs facteurs d'abord entre les paramètres physico-chimiques de l'AMP et des Zones Non Protégées à l'Exploitation Halieutique (ZNPEH), ensuite la composition, la nature des peuplements et les indicateurs bioécologiques des deux sites et enfin la distribution spatiale des espèces.

\section{Matériel et méthode}

\section{Présentation de la zone d'étude}

L'AMP se situe dans la commune de Joal-Fadiouth dans la région de Thiès au Sénégal (Figure 1).

Elle est située au cœur d'une zone où d'importants phénomènes d'upwelling se développent en saison froide et où les apports en matières organiques des fleuves Saloum et Gambie abondent notamment en saison des pluies (Roy, 2011). Pour la pêche l'année est découpée en quatre saisons. La saison froide (SF) correspondant à la période sèche et dure de novembre à mai. Cette période est caractérisée par des eaux de surfaces froides de 16 à $18^{\circ} \mathrm{C}$ et riches en sels nutritifs de l'ordre de 35,5 à 36\%. La transition saison froidesaison chaude (STFC) est la période de passage de la saison froide en saison chaude. Cette période est relativement courte et s'effectue entre mai et juin. Les eaux chaudes tropicales recouvrent progressivement les eaux froides. La saison chaude (SC) s'installe à partir de juillet-août, et est consécutive au retrait des alizés. Elle est marquée par une couche superficielle isotherme de $25^{\circ}$ à $27^{\circ} \mathrm{C}$ et homogène, dont l'épaisseur varie entre 20 et 60 mètres et correspond à la saison des pluies. La transition saison chaude-saison froide (STCF) : Cette période (octobre-décembre) correspond au retrait des eaux chaudes et des précipitations mais également à l'installation des alizés ( Sarr et al, 2018). La mise en place des alizés induisant l'upwelling côtier ou la dislocation de la thermocline sous l'effet des ondes internes pourraient en être la cause $(B a, 2017)$. 
La zone protégée comprend les dépendances maritimes de la commune de Joal-Fadiouth, un bras de mer, ainsi, qu'un important réseau de mangroves (Tableau 2). La surveillance totale de la réserve est sous la responsabilité de la Direction des Aires Marines Communautaires Protégées (DAMCP) du Ministère de l'Environnement et du Développement Durable.

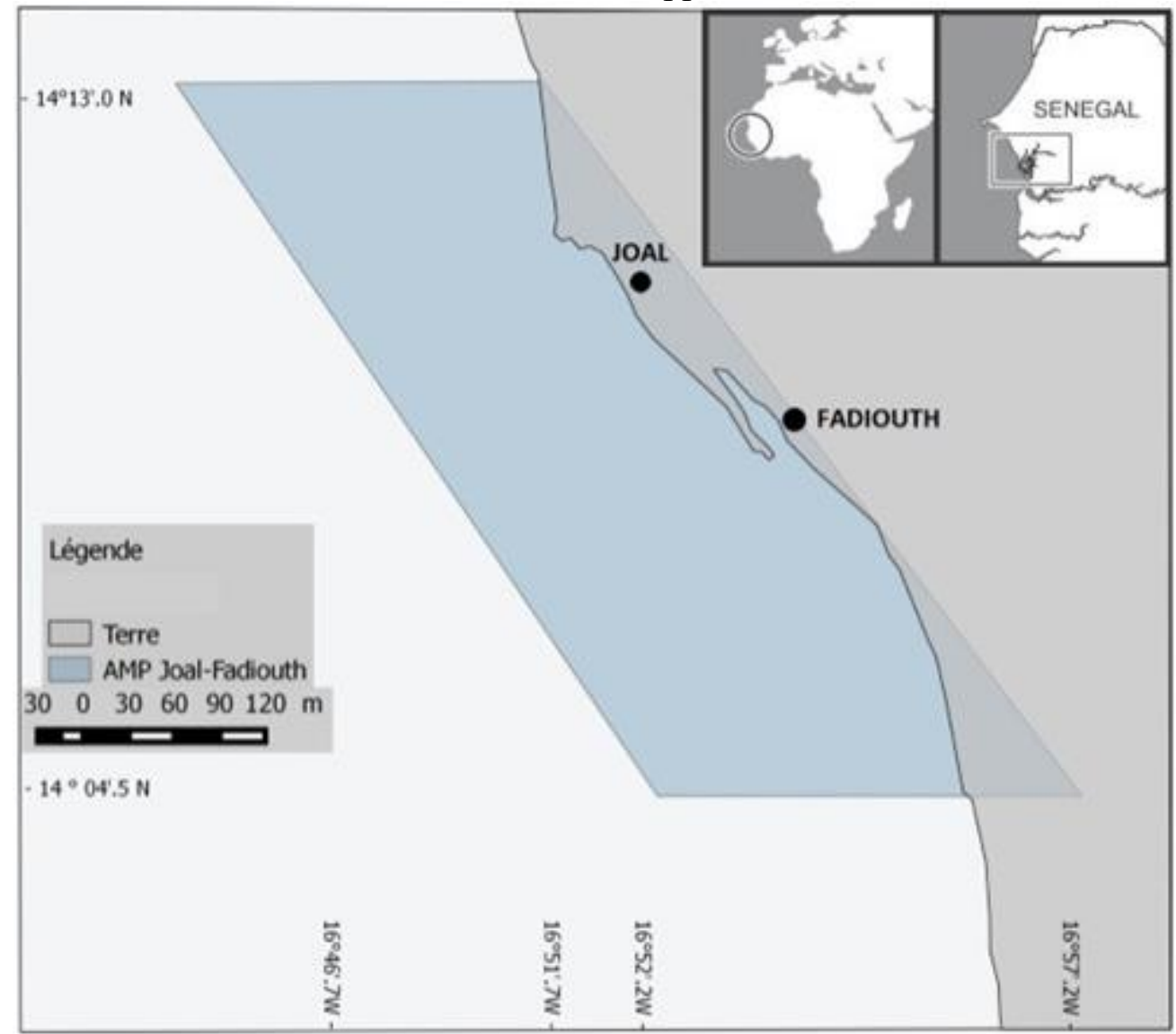

Figure 2 : Localisation de la commune de Joal-Fadiouth (AMP, 2005)

Tableau 1. Délimitation de l'AMP de Joal-Fadiouth

\begin{tabular}{|c|c|c|c|c|c|}
\hline Zones de Relevés & \multicolumn{2}{|l|}{ Côte } & \multicolumn{2}{|l|}{ Mer } & $\begin{array}{l}\text { Superficie } \\
(\mathbf{k m 2})\end{array}$ \\
\hline Coordonnées & Latitude & Longitude & Latitude & Longitude & \multirow{3}{*}{174} \\
\hline 1 & $14^{\circ} 04^{\prime} .5 \mathrm{~N}$ & $16^{\circ} 46^{\prime} .7 \mathrm{~W}$ & $14^{\circ} 04^{\prime} .5 \mathrm{~N}$ & $16^{\circ} 51^{\prime} .7 \mathrm{~W}$ & \\
\hline 2 & $14^{\circ} 13^{\prime} .0 \mathrm{~N}$ & $16^{\circ} 52^{\prime} .2 \mathrm{~W}$ & $14^{\circ} 13^{\prime} .0 \mathrm{~N}$ & $16^{\circ} 57^{\prime} .2 \mathrm{~W}$ & \\
\hline
\end{tabular}

\section{Matériel}

Dans le cadre de cette étude, différents matériels ont été utilisés. Pour la mesure des paramètres physico-chimiques, le matériel utilisé est composé: 
- une sonde multi-paramètres, YSI pour effectuer les mesures de la teneur en oxygène dissous et la température;

- un réfractomètre (de précision à 1 unité de salinité) pour la mesure de la salinité,

- un sondeur à main pour mesurer la profondeur des différentes zones ;

- un GPS Garmin pour prendre les coordonnées géographiques;

- un disque de Secchi à diamètre $10-15 \mathrm{~cm}$ pour mesurer la transparence;

Pour le suivi bioécologique un certain nombre de matériel a été également utilisé :

- un ichtyomètre pour les mensurations (longueur totale);

- une balance commerciale et une balance numérique pour peser respectivement les poissons et les gonades ;

- une fiche de pointage ;

- un guide d'entretien ;

- des bassines et des paniers pour contenir les poissons ;

- un appareil photo pour la prise de vues ;

- un filet (une senne tournante longueur $250 \mathrm{~m}$, hauteur de chute $24 \mathrm{~m}$ et de maille $14 \mathrm{~mm}$ de diamètre) pour capturer les poissons ;

- une pirogue motorisée comme moyen d'embarcation pour la navigation

\section{Méthode}

Méthodologie d'échantillonnage pour la collecte des données à l'intérieur et à l'extérieur de l'AMP pour la pêche expérimentale de la saison froide

Un plan d'échantillonnage aléatoire a été adopté pour la pêche expérimentale. Ainsi, au niveau de l'AMP de Joal-Fadiouth, sur les 40 carrés de 1 mile marin de côté 06 ont été tirés au hasard et sans remise. A l'intérieur de chacun de ces carrés, un coup de pêche est effectué sur une surface bien déterminée d'environ 0,5ha. Cette surface correspond au point de la station de pêche. En plus de ces 06 stations de pêche, 10 autres ont été rajoutées dont 03 dans la lagune de l'AMP contre 07 dans les zones non protégées à l'exploitation halieutique. Enfin, l'intérieur de l'AMP, s'est retrouvé avec 09 stations contre 07 dans les zones non protégées à l'exploitation halieutique (Figure 2). La distance entre chaque station située à l'extérieur et les limites de l'AMP est au minimum d'un kilomètre. Les différentes stations ont été fixées au cours de la campagne de pêche expérimentale.

Ce zonage écologique a été effectué avec la collaboration effective des communautés locales, des agents de la Direction de Pêche Maritime (DPM), de la Direction de Protection et de la Surveillance des Pêches (DPSP), de 
l'AMP et du Centre de Recherche Océanographique Dakar-Thiaroye (CRODT).

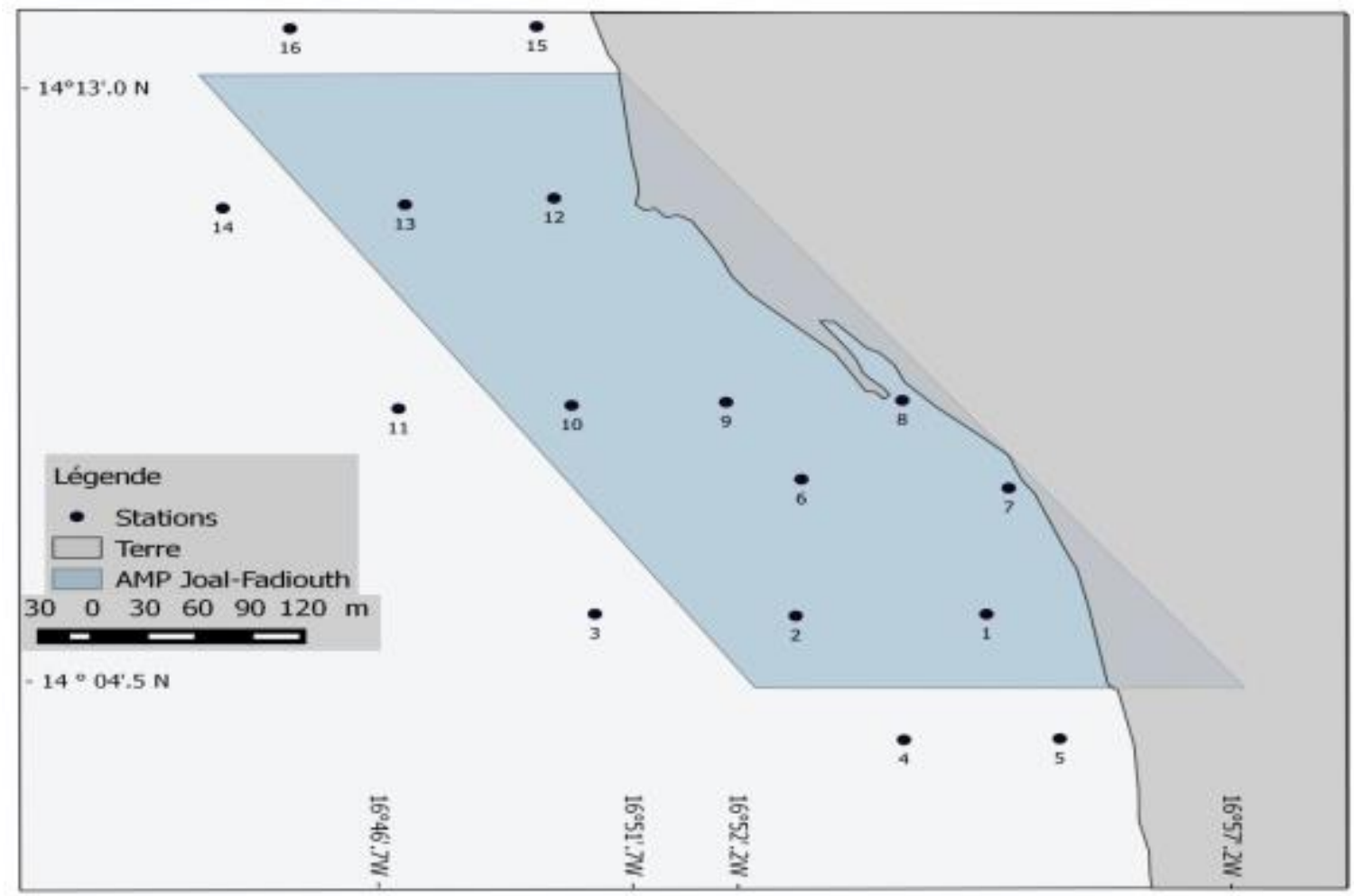

Figure 2 : Localisation des stations échantillonnées dans cette étude.

La senne tournante coulissante a été utilisée lors de la pêche expérimentale. Cette méthode permet d'une part une bonne reproductibilité de l'échantillonnage dans des milieux très variés et d'autre part, une récolte du matériel biologique en bon état (Simier et al., 2004). L'engin a été utilisé sans recherche de banc de poisson, et avec, à chaque fois, la même équipe de pêcheurs et le même mode opératoire. La senne tournante coulissante permet de considérer un coup de pêche comme une unité d'effort stable, autorisant ainsi la comparaison directe entre les coups de pêche. D'une longueur totale de $250 \mathrm{~m}$, d'une hauteur de chute de $24 \mathrm{~m}$ et des mailles de $14 \mathrm{~mm}$, la senne tournante coulissante balaie une surface estimée à 0,5 ha par coup de pêche d'environ 25 minutes.

Les poissons récoltés sont triés par espèce, puis par individu de l'ensemble ou d'une fraction importante (30 individus au maximum) sont pesés (poids total frais, au gramme près) et mesurés au millimètre près. Ainsi, la longueur totale (Lt) est mesurée avec un ichtyomètre du point le plus en avant de la tête, bouche fermée, jusqu'à l'extrémité de la queue bien rabattue. Les poissons pesés sont éviscérés pour déterminer le sexe et le stade de 
maturité sexuel. A l'exploitation des données, les espèces sont enfin groupées par catégorie écologique et par catégorie trophique respectivement selon la classification établie par Albaret., (1999) et à partir des données de Fishbase et de la littérature.

Tableau 2. Guilde des catégories écologiques et trophiques

\begin{tabular}{l|l}
\hline Code & Description \\
\hline Catégories écologiques \\
\hline Es & Estuarienne strict \\
\hline $\mathrm{Em}$ & Estuarienne marine \\
\hline $\mathrm{ME}$ & Marine estuarienne \\
\hline $\mathrm{Ma}$ & Marine accessoire \\
\hline Mo & Marine occasionnel \\
\hline Ms & Marine strict \\
\hline Catégories trophiques \\
\hline he-de & Herbivore détritivore ou brouteur \\
\hline he-ph & Herbivore à prédominance phytoplanctonophage ou microphytophage \\
\hline p1-zo & Prédateur de premier niveau, zooplanctonophagie dominante \\
\hline p1-bt & Prédateur de premier niveau à prédominance benthophage (mollusques, coquillages, vers) \\
\hline p1-mc & Prédateur de premier niveau macrophage ou généraliste (crustacés, insectes) \\
\hline p2-ge & Prédateur de deuxième niveau généraliste (poissons, crevettes, crabes) \\
\hline p2-pi & Prédateur de deuxième niveau à prédominance piscivore \\
\hline
\end{tabular}

Source : Albaret (1999)

\section{Paramètres physico-chimiques}

Pour apprécier les caractéristiques environnementales des sites, les paramètres suivants sont recueillis dans chaque station d'échantillonnage. Il s'agit de la température $\left({ }^{\circ} \mathrm{C}\right)$, la concentration de l'oxygène dissous $(\%)$, la transparence $(\mathrm{m})$ et la salinité en unité part per thousand (ppt) qui est la quantité en grammes de sels dissous dans un $\mathrm{kg}$ d'eau de mer. La température, l'oxygène dissous ainsi que la salinité, leurs mesures ont été prises à la surface de l'eau et à un niveau un peu plus profond.

\section{Traitement et analyse des données}

La saisie et le traitement des données ont été réalisés à partir des logiciels Excel, XLSTAT et PAST.

\section{Analyse des données}

Cette étude s'est portée au descripteur statistique (la richesse spécifique) et aux indicateurs synthétiques à savoir: les indices de diversité de Shannon-Weaver et d'équitabilité de Pielou. Enfin, l'analyse factorielle des correspondances (AFC) est utilisée pour décrire les peuplements de poissons et leur distribution entre les deux zones de l'étude (AMP et ZNPEH). 
En plus, l'AFC a permis de définir les groupes d'espèces caractérisant chaque milieu.

\section{Indice de diversité de Shannon-Weaver (Sh)}

L'indice de diversité de Shannon-Weaver $\left(\mathrm{H}^{\prime}\right)$ permet de décrire la structure d'une communauté, sa composition en espèces. Elle varie généralement entre 0 (une seule espèce, ou bien une espèce dominant très largement toutes les autres) et 4,5 (diversité très forte). Un indice supérieur à 4,5 mène souvent à une perte de la stabilité de l'écosystème (Gobat et al. 2010). L'indice de diversité de Shannon (Sh) est faible lorsque les individus rencontrés appartiennent tous à une même espèce ou bien quand chaque espèce est représentée par un seul individu ; donc Sh est plus sensible aux espèces rares. Cependant, Sh est optimum quand les individus trouvés sont répartis sur plusieurs espèces, ce qui correspond à un peuplement stable, non soumis à l'action des facteurs abiotiques ou facteurs de pollution (Ndiaye, 2014).

$$
H^{\prime}=-\sum_{i=1}^{s} p i \log _{2} p i
$$

- $\quad \mathrm{H}^{\prime}$ : c'est l'indice de diversité de Shannon ;

- $\quad i: c^{\prime}$ 'est une espèce ;

- $\quad p i$ : C'est la proportion de l'espèce $\boldsymbol{i}$ par rapport au nombre total d'espèces

(S) dans le milieu d'étude (ou richesse spécifique du milieu), qui se calcule de la façon suivante :

$p(i)=n i / N$;

- $\quad n i$ : C'est le nombre d'individus pour l'espèce $\boldsymbol{i}$;

- $\quad \mathrm{N}$ : C'est l'effectif total (les individus de toutes les espèces).

Autrement dit, l'indice de diversité de Shannon varie de 0 (une seule espèce, ou bien une espèce dominant très largement toutes les autres) à $\ln (\mathrm{S})$ (toutes les espèces ont la même abondance). Cependant, l'utilisation de l'indice de Shannon présente un inconvénient car les valeurs prises par $\mathrm{H}^{\prime}$ dépendent à la fois de la richesse et de la répartition des effectifs entre les différentes espèces.

\section{Indice d'équitabilité de Piélou}

L'indice d'équitabilité de Piélou est le rapport entre la diversité spécifique observée (Sh) et la diversité maximale théorique (Shmax) pouvant être obtenue avec le même nombre d'espèces (Frontier et Pichod-Viale, 1991). L'équitabilité de Pielou dérive de l'indice de diversité spécifique et consiste à comparer la diversité de Sh à sa valeur maximale. 


$$
J^{\prime}=H^{\prime} / H^{\prime}{ }_{\max }
$$

$\mathbf{J}^{\prime}=$ indice d'équitabilité,

$H^{\prime} \max =\log \mathrm{S}$ ( $\mathrm{S}=$ nombre total d'espèces).

L'indice d'équitabilité permet de mesurer la répartition des individus au sein des espèces, indépendamment de la richesse spécifique mais permet également d'exprimer la dominance d'une espèce. Sa valeur varie de 0 (dominance d'une des espèces) à 1 (équi-répartition ou les espèces sont équi-fréquentes, donc leur abondance est identique) (Gray et al. 1990).

\section{Distribution spatiale des espèces dans les sites}

Le logiciel XLSTAT est utilisé pour analyser la répartition des espèces dans les deux sites. Cette étude de la répartition des espèces permettra de voir l'arrangement spatial et l'abondance des espèces dans les différents milieux.

\section{Résultats}

\section{Etude comparative des paramètres physico-chimiques}

Chaque coup de pêche est accompagné d'une description de l'environnement aquatique de la station correspondante : salinité en unité part per thousand (ppt), la température en degrés Celsius $\left({ }^{\circ} \mathrm{C}\right)$, transparence en mètre $(\mathrm{m})$ et l'oxygène dissous en $\mathrm{mg} / \mathrm{l}$. En complément, la profondeur à l'emplacement du coup de pêche est mesurée ainsi la nature du fond déterminée. Ces fonds sont : rocheux, gravillonneux, sablo-coquillers, sablovaseux-coquillers, sable. La salinité varie avec une moyenne de 35,27 ppt dans l'Aire Marine Protégée, alors que dans les zones non protégées à l'exploitation halieutique, elle est en moyenne de 34,78 ppt. La transparence est très peu variable $(2,69 \mathrm{~m}$ à l'aire marine protégée et $3,59 \mathrm{~m}$ en zone hors de l'aire marine protégée) et la différence de l'écart type inter sites n'est pas significative $(0,3 \mathrm{~m})$. La température dans l'aire marine protégée est avec une moyenne de $24,81^{\circ} \mathrm{C}$ contre $25,14^{\circ} \mathrm{C}$ dans les zones non protégées à l'exploitation. Cependant, on peut constater une baisse de température dans l'aire marine protégée dans les stations 6, 9 et 12 . Compris en moyenne entre 90,65\% dans l'aire marine protégée et $105,87 \%$ dans les zones non protégées à l'exploitation halieutique, le pourcentage de saturation en oxygène dissous a une différence d'écart type de 6,39\% (Tableau 3). 
Tableau 3 : Paramètres physico-chimiques des deux sites.

\begin{tabular}{|c|c|c|c|c|c|}
\hline Paramètres & Sites & $\begin{array}{c}\text { Nombre } \\
\text { observation }\end{array}$ & Moyenne & $\begin{array}{l}\text { Ecart } \\
\text { type } \\
\text { moyen }\end{array}$ & $\begin{array}{l}\text { Différence } \\
\text { de l'écart }\end{array}$ \\
\hline \multirow{2}{*}{$\begin{array}{ll}\text { Salinité } \\
\text { (ppt) }\end{array}$} & AMP & 09 & 35,27 & 0,58 & \multirow[t]{2}{*}{ $\pm 1,35$} \\
\hline & ZNPEH & 07 & 34,78 & 1,93 & \\
\hline \multirow{2}{*}{$\begin{array}{l}\text { Transparence } \\
\text { (m) }\end{array}$} & AMP & 09 & 2,69 & 1,15 & \multirow[b]{2}{*}{ $\pm 0,3$} \\
\hline & ZNPEH & 07 & 3,59 & 0,85 & \\
\hline \multirow{2}{*}{$\begin{array}{l}\text { Température } \\
\left({ }^{\circ} \mathrm{C}\right)\end{array}$} & AMP & 09 & 24,81 & 0,85 & \multirow[b]{2}{*}{ $\pm 1,6$} \\
\hline & ZNPEH & 07 & 25,14 & 2,45 & \\
\hline \multirow{2}{*}{$\begin{array}{l}\text { Oxygène } \\
\text { dissous (\%) }\end{array}$} & AMP & 09 & 90,65 & 16 & \multirow[b]{2}{*}{ $\pm 6,39$} \\
\hline & ZNPEH & 07 & 105,87 & 9,61 & \\
\hline \multirow{2}{*}{$\begin{array}{l}\text { Profondeur } \\
\text { (m) }\end{array}$} & AMP & 09 & 3,36 & 1,54 & \multirow[b]{2}{*}{ $\pm 0,3$} \\
\hline & ZNPEH & 07 & 5,2 & 1,84 & \\
\hline
\end{tabular}

La figure 3, montre des variations des paramètres physico-chimiques des stations entre l'aire marine protégée et les zones non protégées à l'exploitation halieutique.

En résumé, il n'y a pas une différence significative des paramètres environnementaux qui puissent favoriser grandement la distribution des espèces dans une seule zone. 


\begin{tabular}{|c|c|c|c|c|c|}
\hline $\begin{array}{l}7 \\
6 \\
5 \\
4 \\
3 \\
2 \\
1 \\
0\end{array}$ & 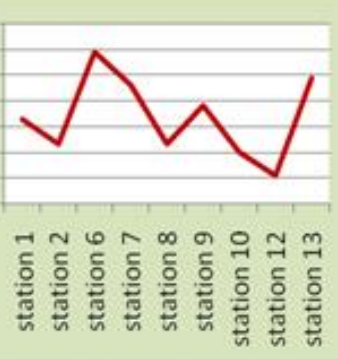 & $\begin{array}{l}\text { Profondeur } \\
\text { des stations } \\
\text { de l'AMP(m) }\end{array}$ & $\begin{array}{l}8 \\
7 \\
6 \\
5 \\
4 \\
3 \\
2 \\
1 \\
0\end{array}$ & 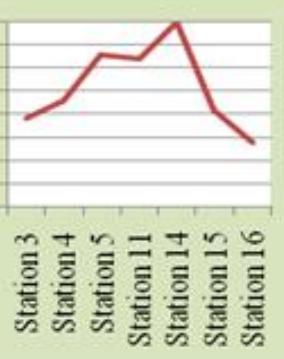 & $\begin{array}{l}\text { Profond } \\
\text { eur (m) } \\
\text { des } \\
\text { stations } \\
\text { des } \\
\text { zones } \\
\text { hors de } \\
\text { l'AMP }\end{array}$ \\
\hline $\begin{array}{l}5 \\
4 \\
3 \\
2 \\
1 \\
0\end{array}$ & 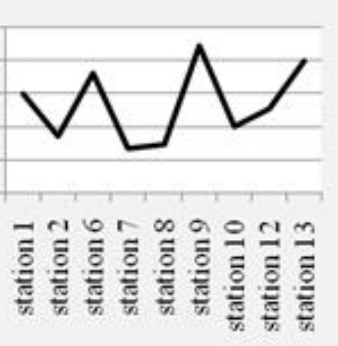 & $\begin{array}{l}\text { - Transparenc } \\
\text { e dans les } \\
\text { stations de } \\
\text { l'AMP (m) }\end{array}$ & $\begin{array}{l}5 \\
4 \\
3 \\
2 \\
1 \\
0\end{array}$ & 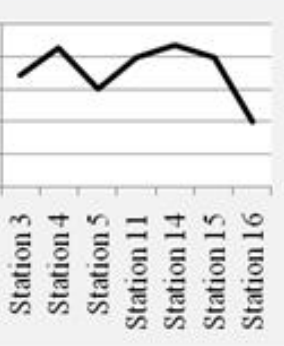 & $\begin{array}{l}\text { Transpare } \\
\text { nce (m) } \\
\text { dans les } \\
\text { stations } \\
\text { des zones } \\
\text { hors } \\
\text { AMP }\end{array}$ \\
\hline $\begin{array}{r}25 \\
20 \\
15 \\
10 \\
5\end{array}$ & 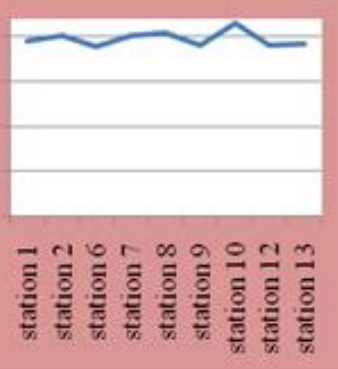 & $\begin{array}{l}\text {-Temperature } \\
\text { moyenne au } \\
\text { niveau des } \\
\text { stations de } \\
\text { l'AMP }\end{array}$ & $\begin{array}{l}35 \\
30 \\
25 \\
20 \\
15 \\
10 \\
5 \\
0\end{array}$ & 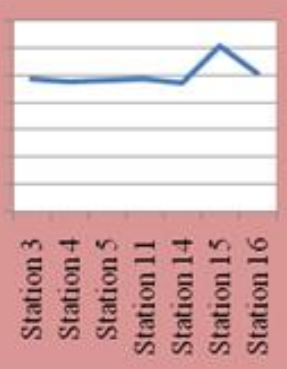 & $\begin{array}{l}\text { - Températ } \\
\text { ure } \\
\text { moyenne } \\
\text { auniveau } \\
\text { des } \\
\text { stations } \\
\text { des zones } \\
\text { hors AMP }\end{array}$ \\
\hline $\begin{array}{r}40 \\
35 \\
30 \\
25 \\
20 \\
15 \\
10 \\
5\end{array}$ & 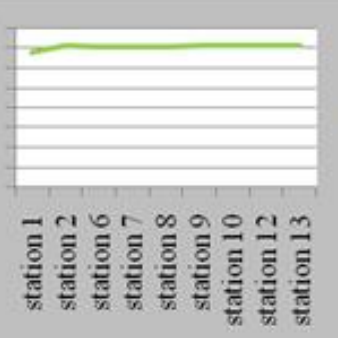 & $\begin{array}{l}\text { Salinité } \\
\text { moyenne au } \\
\text { niveau des } \\
\text { stations de } \\
\text { l'AMP }\end{array}$ & $\begin{array}{r}40 \\
30 \\
20 \\
10 \\
0\end{array}$ & 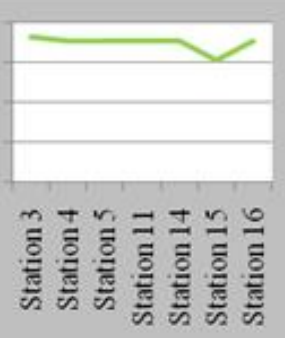 & $\begin{array}{l}\text { Salinité } \\
\text { moyenne } \\
\text { au niveau } \\
\text { des } \\
\text { stations } \\
\text { des zones } \\
\text { hors AMP }\end{array}$ \\
\hline $\begin{array}{r}140 \\
120 \\
100 \\
80 \\
60 \\
40 \\
20 \\
0\end{array}$ & 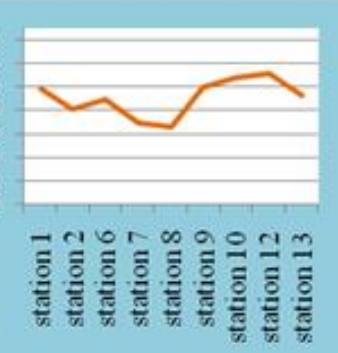 & $\begin{array}{l}\text { Pourcentage } \\
\text { moyen de } \\
\text { l'oxygène } \\
\text { dissous au } \\
\text { niveau des } \\
\text { stations de } \\
\text { l'AMP }\end{array}$ & $\begin{array}{r}140 \\
120 \\
100 \\
80 \\
60 \\
40 \\
20 \\
0\end{array}$ & 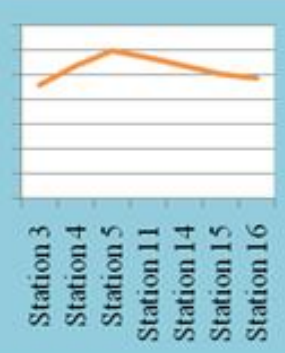 & $\begin{array}{l}\text { Pourcenta } \\
\text { ge moyen } \\
\text { de } \\
\text { l'oxygène } \\
\text { dissous au } \\
\text { niveau des } \\
\text { stations } \\
\text { des zones } \\
\text { hors AMP }\end{array}$ \\
\hline
\end{tabular}

Figure 3 : Variations des paramètres physico-chimiques entre l'AMP et les ZNPEH. 
Nous pouvons ainsi déduire que les paramètres environnementaux dans l'aire marine protégée sont similaires aux zones non protégées à l'exploitation halieutique. Par conséquent, les éventuelles différences de peuplements ne s'expliquent pas par la variabilité des paramètres physicochimiques.

\section{Présentation des indicateurs bioécologiques}

Les indicateurs de biodiversité sont des paramètres de mesure indispensable pour suivre la dynamique de la diversité marine, côtière, agricole et sauvage. Ils fournissent ainsi des données nécessaires pour l'élaboration et l'évaluation des politiques de protection de la biodiversité (Tableau 4).

\begin{tabular}{|c|c|c|c|c|c|c|c|}
\hline \multicolumn{5}{|l|}{9 Diversity } & \multicolumn{2}{|c|}{\begin{tabular}{l|l}
$口$ & 口 \\
\end{tabular}} & \multirow[t]{2}{*}{$x$} \\
\hline & Zone AMP & Zone hors AMP & & & & & \\
\hline Taxa_S & 35 & 18 & & & & & \\
\hline Individuals & 1908 & 119 & & & & & \\
\hline Dominance_D & 0,3715 & 0,1053 & & & & & \\
\hline Simpson_1-D & 0,6285 & 0,8947 & & & & & \\
\hline Shannon_H & 1,41 & 2,501 & & & & & \\
\hline Evenness_e $\mathrm{e}^{\wedge} \mathrm{H} / \mathrm{S}$ & 0,1171 & 0,6775 & & & & & \\
\hline Brillouin & 1,381 & 2,277 & & & & & \\
\hline Menhinick & 0,8013 & 1,65 & & & & & \\
\hline Margalef & 4,501 & 3,557 & & & & & \\
\hline Equitability_J & 0,3967 & 0,8653 & & & & & \\
\hline Fisher_alpha & 6,086 & 5,895 & & & & & \\
\hline Berger-Parker & 0,499 & 0,2101 & & & & & \\
\hline$\Gamma$ Bootstrap & ( $95 \%$ confi & $x$ & 㗨索 & 思 & & & \\
\hline
\end{tabular}

Tableau 4 : les indices de diversité des deux sites

\section{Comparaison de richesse spécifique}

Durant la période de la saison froide 2015 et pour l'ensemble des deux sites d'études, une campagne de pêche expérimentale a été réalisée avec 16 relevés hydrologiques et 16 coups de pêche. Regroupées en 24 familles, les 38 espèces de poissons dont 35 à l'aire marine protégée et 18 dans les zones non protégées à l'exploitation halieutique ont un effectif de 2027 individus Parmi les 24 familles les mieux représentées dans l'aire marine protégée en nombre d'espèces dans l'AMP sont :

* Sparidae et les Haemulidae avec 04 espèces chacune soit 11,43\% respectivement; 
Carangidae et les Tetraodontidae font chacune 3 espèces soit 8,57 \% ;

- Cichlidae, Gerreidae, Mugilidae, Serranidae font chacune 02 espèces soit $5,71 \%$.

Les autres familles (16) font chacune 01 espèce soit 2,85 \% (Figure 4).
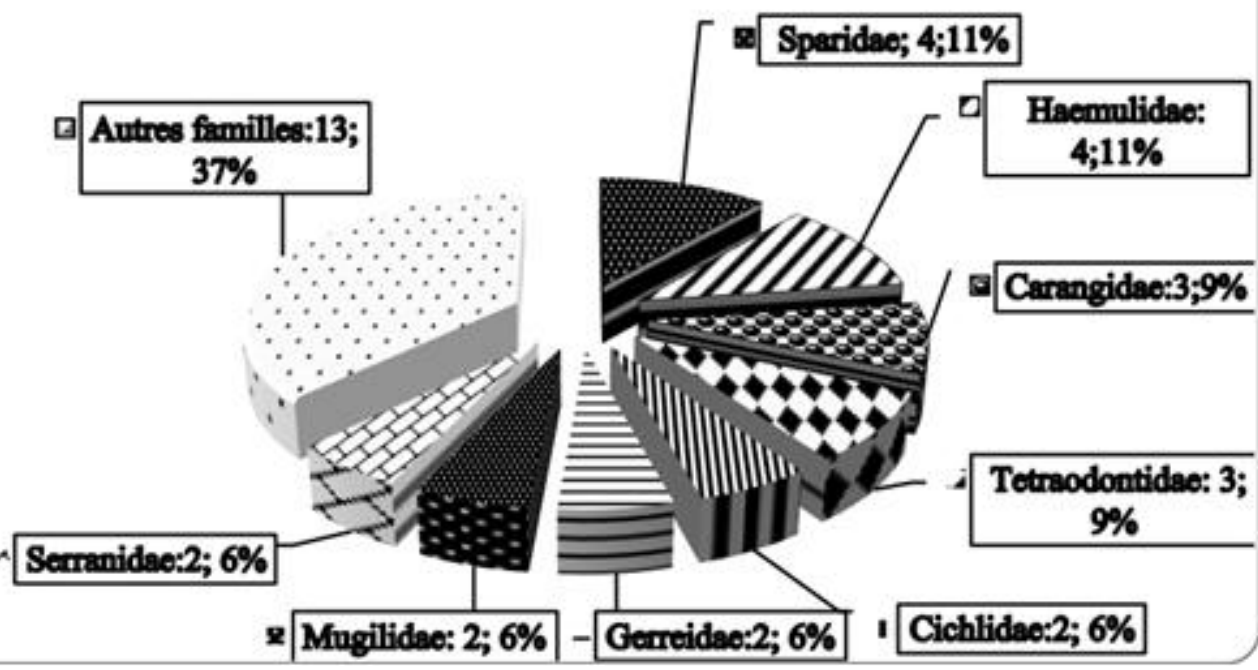

Figure 4 : Pourcentage des familles les plus représentées dans l'AMP

Par contre dans les zones non protégées à l'exploitation halieutique, les familles les mieux représentées parmi les 18 en nombre d'espèces sont :

Gerreidae, Sparidae, Tetraodontidae chacune 02 espèces soit $11,11 \%$. Le reste des 12 autres familles font chacune 01 espèce soit $5,56 \%$ de l'effectif total (Figure 5). 
Autres familles; 11; $61 \%$

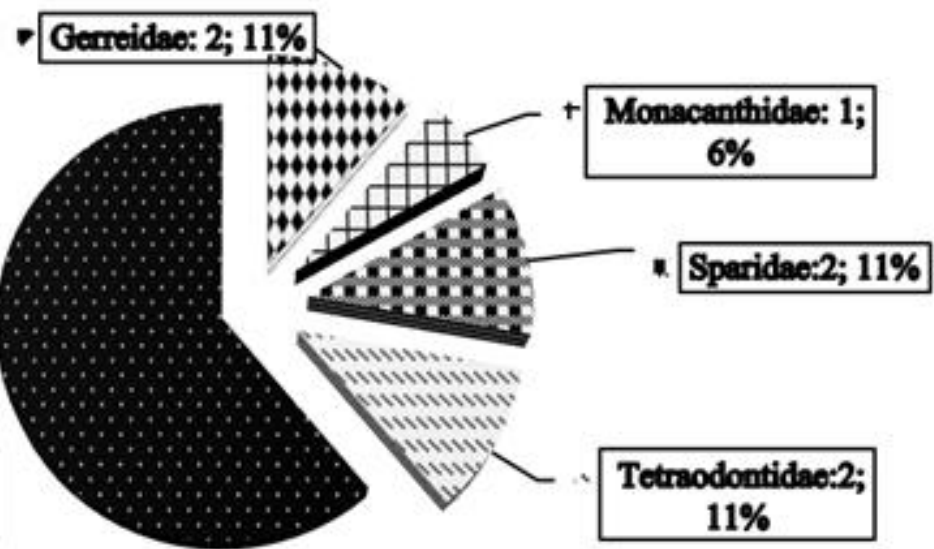

Figure 5 : Pourcentage des familles les plus représentées dans les ZNPEH

En effet, en comparant les deux sites, nous constatons que dans l'aire marine protégée les familles Sparidaes et Haemulidaes sont les plus représentées en nombre d'espèces (04) soit 10,53\%. Cependant, dans les zones non protégées à l'exploitation halieutique, ce sont les familles Gerreidaes, Monacanthidaes, Tetraodontidaes qui sont les mieux représentées avec le même nombre d'espèces (02) soit 10,53 \% respectivement.

La richesse spécifique totale des deux sites de l'étude est composée de 38 espèces dont 35 dans l'aire marine protégée contre 18 dans les zones non protégées à l'exploitation halieutique. Toutefois, 15 espèces soit $39,47 \%$ de l'effectif total (38 espèces) sont communes aux deux zones. Cependant, les espèces les plus représentées en termes d'individus à l'AMP sont :

- Eucinostomus melanopterus 952 individus soit 50\% de l'effectif total ;

- Liza dumerilii 660 individus soit 34,59\%.

Les autres espèces sont moins représentées (Figure 6). 


\section{Pourcentage des espèces les plus représentées à l'AMP}

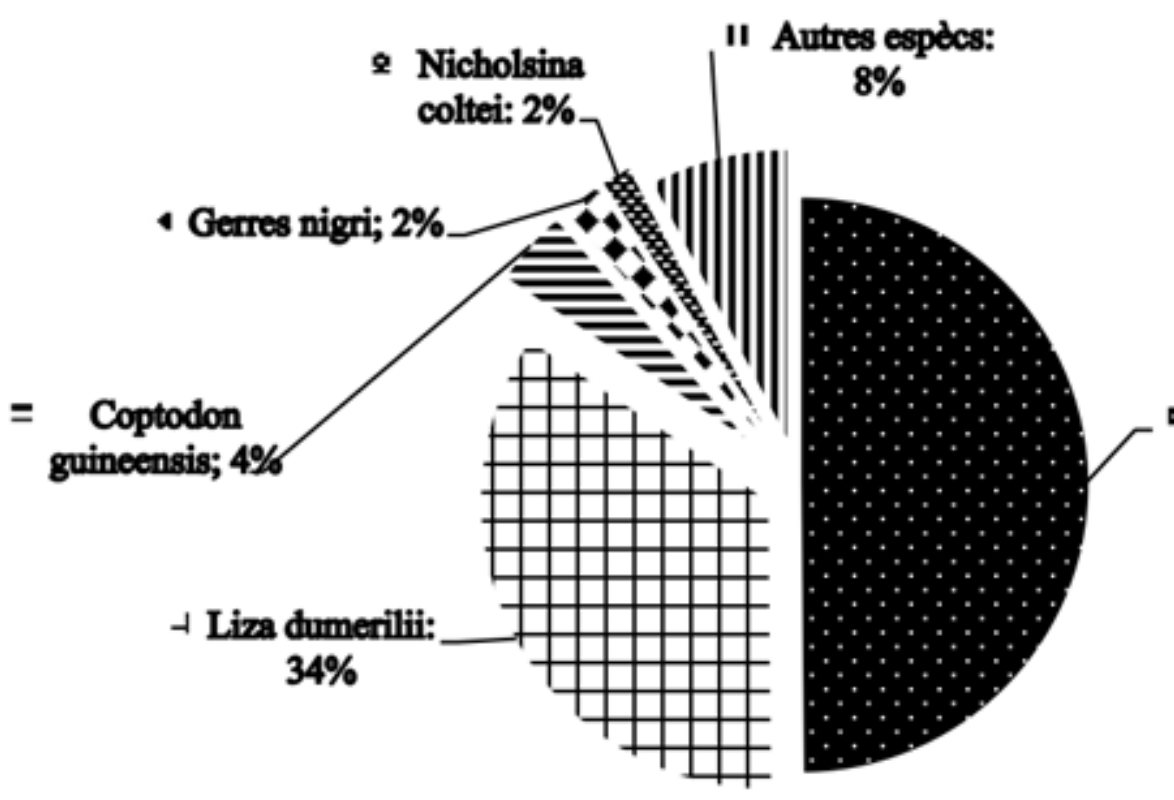

- Eucinostomus melanopterus: $50 \%$

Figure 6: Pourcentage des espèces les plus représentées dans l'AMP

Ces espèces sont caractéristiques des estuaires, des lagunes et des eaux saumâtres ce qui correspond à la zone AMP.

Par contre dans les zones non protégées à l'exploitation halieutique, les 05 espèces (Figure 7) les plus représentées sont :

- Nicholsina collettei avec 25 individus soit $21 \%$;

- Pseudupenaeus prayensis avec 15 individus soit $13 \%$;

- Stephanolepis hispidus avec 15 individus soit $13 \%$;

- Hemiramphus brasiliensis avec 11 individus soit 9,24\% ;

- Sardinella maderensis avec 09 individus soit 7,56 \%. 


\section{Pourcentage des 05 espèces les plus représentées dans les ZNPEH}

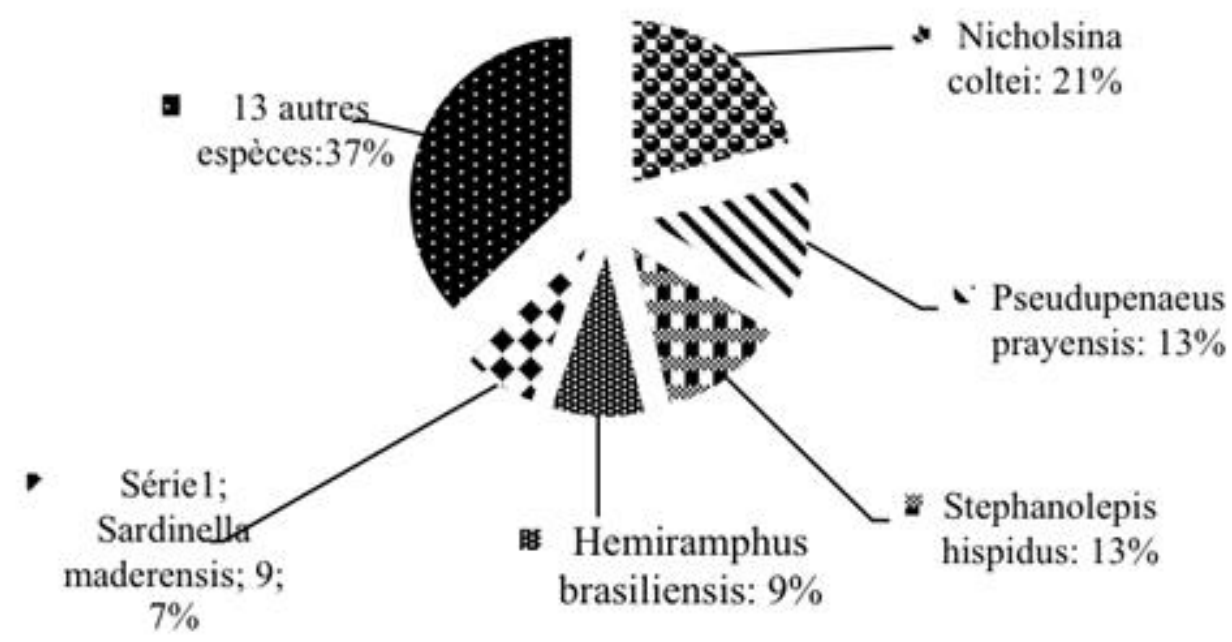

Figure 7: Pourcentage des espèces les plus représentées dans les ZNPEH

\section{Collecte de données primaires sur le terrain}

Les interviews semi-structurées de quelques acteurs locaux (le comité de gestion de l'AMP et le groupement des jeunes pêcheurs) en vue d'apprécier la diversité spécifique de la faune ichtyologique ont donné les résultats suivants :

- 37 espèces regroupées en 26 familles dans l'AMP ;

- 17 espèces regroupées dans 14 familles dans les ZNPEH.

La quasi-totalité des acteurs ont soutenu reconnaitre l'importance de l'AMP qui pour eux constitue une zone de fraie et de nourricerie pour améliorer les ZNPEH. Ils sont tous conscient des textes et lois régissant les différentes zones. Cependant, pour l'AMP, les pêcheurs relèvent des difficultés liées à la reconnaissance des limites de l'AMP et de ses sous zones où il n'y a plus de balise (zone à pêche responsable et la zone totalement interdite à la pêche). L'éloignement entre les balises de délimitation de l'AMP constitue un réel problème pour les pêcheurs.

En résumé, les résultats de ces entretiens apparaissent comme suit :

\section{Etude comparative des indicateurs synthétiques}

L'analyse avec le logiciel Past a permis d'obtenir un tableau avec différents indices cependant nous nous focaliserons aux indicateurs synthétiques en l'occurrence l'indice de Shannon-Weaver et Pielou. 


\section{Indices de diversité de Shannon-Weaver comparés (Sh)}

L'indice de diversité de Shannon-Weaver (Sh) nous permet de décrire la dynamique des peuplements ichtyologiques dans les deux sites (AMP et ZNPEH).

Ainsi, l'analyse de cet indicateur biologique avec le logiciel Past a permis d'avoir respectivement les valeur 1,41 et 2,50 pour la faune ichtyologique de l'AMP de Joal-Fadiouth et celle de la zone non protégée contigüe. Une représentation qui témoigne d'une plus grande diversité du peuplement de poisson au niveau des deux zones. Du point de vu d'importance de la biodiversité, le plus grand nombre d'espèces de poissons rencontrées dans l'AMP est presque le double de la richesse spécifique de la zone non protégée à l'exploitation halieutique. En plus, les individus trouvés dans l'AMP sont répartis sur plusieurs espèces ce qui correspond à un peuplement stable, non soumis à l'action des facteurs abiotiques ou facteurs de pollution et ceci est confirmé par les résultats de Ndiaye, (2014).

\section{Indices d'équitabilité de Pielou comparés}

L'indice d'équitabilité de Pielou connait des variations entre l'AMP $(0,3967)$ et les zones non protégées à l'exploitation halieutique $(0,8653)$. Ces résultats montrent qu'il y a une forte dominance de certaines espèces dans l'AMP comparée aux zones non protégées à l'exploitation halieutique où l'indice tend vers 1 (équirépartition ou les espèces sont équi-fréquentes, donc leur abondance est identique). Et, en se référant au tableau 5 et 6 (nombre d'individus à l'AMP et dans la zone hors AMP), nous remarquons bien à l'AMP, cette forte représentativité des espèces E. melanopterus $(49,90 \%), L$. dumerilii $(34,59 \%)$ alors que dans les ZNPEH ce sont les espèces $N$. coltei (21\% de l'effectif total), $P$. prayensis et $S$. hispidus (12\%) qui sont les plus abondantes. Cette différence entre les indices d'équitabilité de Piélou dans l'AMP et dans les zones non protégées à l'exploitation halieutique, s'explique par une plus grande abondance de stock d'espèces dans la réserve protégée.

\section{Etude comparative de la nature des peuplements entre les deux sites}

Au niveau de l'AMP et dans les zones non protégées à l'exploitation halieutique, les différentes catégories écologiques sont identifiées en fonction de l'affinité marine ou continentale des espèces. Enfin, les catégories trophiques sont également identifiées suivant le régime alimentaire des espèces.

\section{Catégories écologiques dans l'AMP}

Le peuplement observé dans l'AMP est composé de 24 familles avec 35 espèces appartenant à 05 des 08 catégories écologiques définies par Albaret (1999), à savoir les espèces Marines occasionnelles (Mo) avec 10 espèces soit 
28,57\%, les Marines d'origine estuarienne (Me) également avec 10 espèces soit $28,57 \%$, les Estuariennes d'origine marine $(\mathbf{E m})$ sont 06 espèces soit 17,14\%, les marines accessoires (Ma) avec 05 espèces soit 14,29\% et les Estuariennes strictes (Es) avec 04 espèces soit 11,43\% (Tableau 5).

Tableau 5 : Catégories écologiques à l'AMP

\begin{tabular}{|l|l|l|}
\hline Nombre d'espèces & Catégories écologiques & Pourcentage (\%) \\
\hline 10 & Mo & 28,57 \\
\hline 10 & Me & 28,57 \\
\hline 6 & Em & 17,14 \\
\hline 5 & Ma & 14,29 \\
\hline 4 & Es & 11,43 \\
\hline
\end{tabular}

Le peuplement de base de l'AMP est constitué par les espèces Mo et Me qui représentent chacun 29\%, soit 58\% des catégories écologiques

\section{Catégories écologiques dans les ZNPEH}

Dans les zones non protégées à l'exploitation halieutique, le peuplement observé est composé de 15 familles avec 18 espèces appartenant à 5 catégories écologiques, à savoir les espèces Marines occasionnelles (Mo) avec 08 espèces soit 44,44\% , les Marines d'origine estuarienne (Me) avec 02 espèces soit $11,11 \%$, les estuariennes d'origine marine $(\mathbf{E m})$ avec 03 espèces soit 16,67\%, les marines accessoires (Ma) avec 04 espèces soit 22,22 et les Estuariennes strictes (Es) avec 01 espèces soit 5,56\% (Tableau 6).

Tableau 6 : Les catégories écologiques au niveau des ZNPEH

\begin{tabular}{|l|l|l|}
\hline Nombre d'espèces & Catégories écologiques & Pourcentage (\%) \\
\hline 8 & Mo & 44,44 \\
\hline 2 & Me & 11,11 \\
\hline 3 & Em & 16,67 \\
\hline 4 & Ma & 22,22 \\
\hline 1 & Es & 5,56 \\
\hline
\end{tabular}

Comme dans l'AMP, les espèces Mo sont les plus représentées, avec 44,4\% et aussi les espèces Es sont les moins représentées (5,5\%).

\section{Analyse comparée des catégories écologiques des deux sites}

En termes de caractéristiques écologiques, le peuplement de poissons de l'AMP et de la zone hors AMP est dominé par les espèces Mo et Me avec respectivement $28,57 \%$ et $44,44 \%$ ensuite $28,57 \%$ et $11,11 \%$.

\section{Catégories trophiques au niveau de l'AMP}

En fonction de leur régime alimentaire, les espèces sont groupées en catégories trophiques comme l'indique le tableau 7. 
Tableau 7 : Pourcentage des catégories trophiques à l'AMP.

\begin{tabular}{|l|l|l|}
\hline Nombre d'espèces & Catégories trophiques & Pourcentage (\%) \\
\hline 1 & p1-zo & 2,86 \\
\hline 4 & p2-pi & 11,43 \\
\hline 4 & he-de & 11,43 \\
\hline 8 & p2-ge & 22,86 \\
\hline 4 & p1-mc & 11,43 \\
\hline 13 & p1-bt & 37,14 \\
\hline 1 & he-ph & 2,86 \\
\hline
\end{tabular}

Avec le tableau 8, nous constatons que les p1-bt sont les plus présents avec $37 \%$ suivi par les p2-ge avec $23 \%$. Au niveau de la zone non protégée hors AMP, c'est aussi pratiquement le même cas de figure qui se présente. Comme le montrent le tableau 8 et la figure 6,37,14\% des espèces appartiennent à la catégorie des prédateurs de premier niveau à prédominance benthophage, mollusques (p1-bt), vers et $23 \%$ représente la catégorie trophique des prédateurs de deuxième niveau à prédominance piscivore $\mathrm{p} 2$ ge).

Tableau 8: Pourcentage des catégories trophiques dans les ZNPEH

\begin{tabular}{|l|l|l|}
\hline Nombre d'espèces & Catégories trophiques & Pourcentage (\%) \\
\hline 1 & p1-zo & 5,56 \\
\hline 3 & p2-pi & 16,67 \\
\hline 1 & he-de & 5,56 \\
\hline 3 & p2-ge & 16,67 \\
\hline 4 & p1-mc & 22,22 \\
\hline 6 & p1-bt & 33,33 \\
\hline
\end{tabular}

Nous constatons que les p1-bt et les p1-mc sont les plus représentatives avec respectivement $33 \%$ et $22 \%$ (Tableau 8 ).

\section{Analyse comparée des catégories trophiques des deux sites.}

Nous constatons que dans les deux milieux, les espèces de la catégorie trophique p1-bt sont les plus représentatives avec 37,14\% à l'AMP et 33,33\% dans les ZNPEH

Le logiciel XLSTAT a permis de faire quelques études comme l'Analyse Factoriel des Correspondances et le test de Khi deux.

\section{Analyse factorielle des correspondances}

$\mathrm{L}$ 'analyse de la répartition des espèces dans un plan à deux axes F1-F2 permet de constater que les peuplements des deux différentes zones sont répartis inégalement, mais avec une forte concentration dans l'AMP. Ainsi, nous pouvons constater que 23 espèces (Eucinostomus melanopterus, Liza dumerilii, Coptodon guineensis etc.) soit $60,52 \%$ de l'effectif total sont plus 
présentes dans l'AMP que dans les zones non protégées à l'exploitation halieutique où on peut voir 06 espèces (Scorpaena stephanica, Trachinocephalus myops, Sardinella maderensis etc.) soit 15,78\%. On peut également constater 09 espèces (Nicholsina coltei, Ephippion guttifer, Pseudupenaeus prayensis etc.) réparties de manière assez similaire entre l'AMP et les zones non protégées à l'exploitation halieutique (Figure 8).

$\mathrm{Au}$ regard de la configuration de la répartition des espèces au niveau des deux zones, on constate que plus de la moitié des espèces rencontrées ont une affinité beaucoup plus forte dans l'AMP que dans les zones non protégées à l'exploitation halieutique.

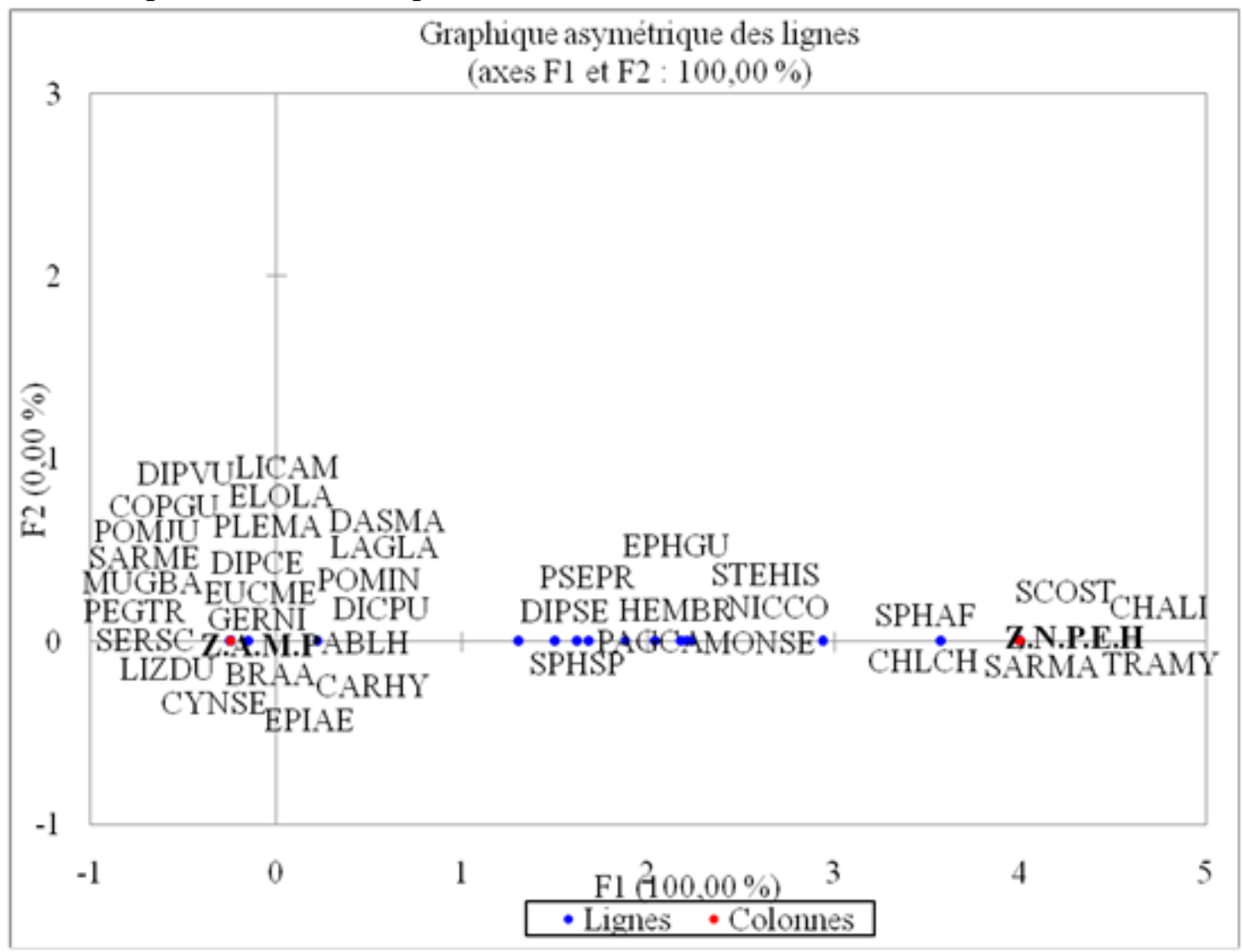

Figure 8 : Distribution des espèces dans l'AMP et dans les ZNPEH

\section{Le test de Khi-deux}

Le test de Khi-deux est utilisé pour tester l'hypothèse nulle d'absence de relation entre deux variables catégorielles. On peut également dire que ce test vérifie l'hypothèse d'indépendance de ces variables.

Ainsi avec le test de Khi-deux d'indépendance entre les lignes et les colonnes montre que : 
Nous avons les hypothèses :

$\mathrm{H} 0$ : les lignes et les colonnes sont indépendantes alors les individus des deux zones sont différents.

Ha : Il existe un lien entre les lignes et les colonnes du tableau c'est-àdire les individus des deux zones sont identiques et que toute différence observée est due à une variation aléatoire.

Etant donné que la p-value calculée est inférieure au niveau de signification alpha $=0,05$, on doit rejeter l'hypothèse nulle $\mathrm{H} 0$, et retenir l'hypothèse alternative Ha.

Le risque de rejeter l'hypothèse nulle $\mathrm{H} 0$ alors qu'elle est vraie est inférieur à 0,01\% (Tableau 9).

En résumé, nous pouvons déduire que les 02 variables (individus AMP et ZNPEH) sont identiques car il n'existe pas une différence significative car le seuil de risque 5\% est supérieur au p-value qui est égale à 0,0001

Tableau 9: Test de Khi-deux d'indépendance entre les zones

\begin{tabular}{|l|l|}
\hline Khi $^{2}$ (Valeur observée) & 1017,490 \\
\hline Khi $^{2}$ (Valeur critique) & 52,192 \\
\hline DDL & 37 \\
\hline p-value & $<0,0001$ \\
\hline Alpha & 0,05 \\
\hline
\end{tabular}

\section{Discussions}

L'objectif de cette étude est d'évaluer l'efficacité de la gestion de l'AMP par une approche biologique (diversité spécifique de peuplement ichtyologique) comparée avec les zones non protégées à l'exploitation halieutique durant la saison hydro-climatique froide 2015.

Ainsi, avant toute comparaison de ces peuplements, il est nécessaire, dans un premier temps, de vérifier que les conditions hydro-climatiques subies par ces peuplements sont de même ordre pour éliminer un éventuel effet de certaines variables de l'environnement.

Ensuite nous avons abordé la composition et la nature du peuplement ;

Enfin la comparaison des indicateurs synthétiques (Shannon et Pielou) des deux milieux est faite pour déterminer la répartition spatiale des espèces dans les deux zones.

\section{Paramètres physico-chimiques}

Les résultats des paramètres environnementaux: température $\left(24,81^{\circ} \mathrm{C}\right.$ dans $1^{\prime} \mathrm{AMP}$ et $25,14^{\circ} \mathrm{C}$ dans les ZNPEH), oxygène dissous $(90,65 \%$ dans l'AMP et 105,87 \% dans les ZNPEH) et la salinité (comprise entre 35,27 ppt dans l'AMP et 34, 78 ppt dans les ZNPEH) sont comparables à ceux trouvés par Mbengue (2016) lors de l'état de référence de l'AMP de JoalFadiouth. 
De même, Thiam et al., (2012) ont aussi obtenu les mêmes résultats au niveau des zones potentielles pour l'implantation de récifs artificiels à Ngaparou (Petite Côte du Sénégal).

D'après Diouf, (1996), les gammes de températures $\left(24^{\circ} \mathrm{C}-31^{\circ} \mathrm{C}\right)$, d'oxygène $(65 \%-121 \%)$ et de salinité (29 Practical Salinity Unit - 36 PSU) obtenues sont optimales pour la survie de diverses espèces de poissons vivant dans les zones marines et estuariennes d'Afrique de l'Ouest. Egalement, d'après Morris, (2006), la structure spatiale du peuplement peut être influencée par des paramètres tels que la température, la salinité ou l'oxygène. Or, l'analyse des données environnementales dans l'AMP et dans les ZNPEH montre une différence non significative.

Nous pouvons ainsi déduire que les paramètres environnementaux dans l'aire marine protégée sont similaires aux zones non protégées à l'exploitation halieutique. Les éventuelles différences de peuplements ne s'expliquent pas par la variabilité des paramètres physico-chimiques.

Ainsi, l'AMP et les ZNPEH peuvent être considérés comme des milieux homogènes du point de vue environnement.

Cependant seuls la saisonnalité, les phénomènes de changements climatiques et les actions anthropiques pourraient influencer la baisse de la biodiversité ichtyologique des deux sites.

\section{Nature et composition des peuplements des milieux}

Les espèces inventoriées dans l'AMP et dans les ZNPEH pendant la période froide 2015 ont une forte affinité marine (Mo, ME, Ma, Em, Es). Cependant l'absence des espèces à affinité continentale peut s'expliquer par l'utilisation des sennes de plage qui sont des engins très destructeurs des habitats des poissons côtiers, les nurseries et les aires de recrutement (Hounsounou et Zacharie, 2014). La présence des espèces qui se reproduisent essentiellement en milieu estuarien (Es) est relativement faible. En effet, en saison sèche, l'évaporation et la diminution des apports d'eau douce induisent une augmentation de la salinité dans les zones plus internes des milieux estuariens et lagunaires laissant la possibilité aux catégories à affinité marine de pénétrer plus en amont, dans les secteurs intermédiaires (Chabanne, 2007). Ainsi, le peuplement de l'AMP est majoritairement dominé par les Mo, Me et Ma. Ces résultats révèlent que le peuplement de l'AMP de Joal-Fadiouth est très marin, mais avec la présence d'espèces pouvant se développer aussi bien en zone estuarienne qu'en zone marine (Em et ME). Les Mo et Me sont généralement plus abondantes dans des eaux poly-mixoeuhaline (de 18 à 40) (Chabanne, 2007).

Cependant ces données sur la diversité du peuplement de poissons sont à nuancer car tout dépend de la sélection des engins à utiliser pour la pêche expérimentale. 
Et en comparant ces résultats avec ceux de la saison froide de 2014, on constate une différence nette : 48 espèces en 2014 (Diedhiou, 2015) contre 35 espèces en 2015. Cela s'explique par l'utilisation en 2014 de 03 types d'engins.

Cependant cette richesse spécifique (35 espèces) dans l'AMP pendant la saison froide 2015 est un peu semblable à celui de l'inventaire des ressources halieutiques réalisé en 2006 par WWF WAMER. Cet inventaire en 2006 a fait état de 31 espèces de poissons avec une senne de plage (Plan d'aménagement et de gestion 2014-2018 de l'AMP de Joal-Fadiouth).

En effet, d'après Lobry., (2004), la probabilité de capturer une espèce supplémentaire augmente avec la surface explorée et le nombre de prélèvements effectués.

\section{Indicateurs bioécologiques}

\section{Richesse spécifique}

La richesse spécifique totale de l'AMP de Joal-Fadiouth (35 espèces) est relativement élevée par rapport à Cayar avec 26 espèces (Ndiaye, 2014). La diversité des habitats en mer et la présence de la mangrove expliqueraient la présence de plusieurs espèces dans l'AMP et aux alentours (Diallo, 2008). Cependant, elle reste faible dans les ZNPEH. Cette situation pourrait s'expliquer par l'exploitation accrue de la ressource.

\section{Indice de diversité de Shannon-weaver}

La comparaison des indices de diversité Shannon entre l'AMP et les ZNPEH montre une différence significative. L'indice de diversité de Shannon varie entre 1,41 à l'AMP et 2,501 dans la ZNPEH. Cette différence s'explique par l'existence de mesures de gestion en vigueur dans l'AMP, ce qui n'est pas le cas dans les zones non protégées hors AMP. De telles mesures de gestion ont favorisé la présence de la quasi-totalité des effectifs qui sont représentés par E. melanopterus (49,9\% de l'effectif total) et sur L. dumerilii $(34,59 \%$ de l'effectif total) dans l'AMP. La quasi-totalité des effectifs est concentrée sur E. melanopterus (49,9\% de l'effectif total) et sur L. dumerilii $(34,59 \%$ de l'effectif total) dans l'AMP. Cependant dans les ZNPEH la quasi-totalité des effectifs est concentrée sur $N$. coltei (21\% de l'effectif total) et $P$. prayensis et S. hispidus (12\%). Ainsi, le calcul de ces deux indices de Shannon ont permis de mettre en évidence les différences de structure des communautés qui s'explique soit par l'impact de l'exploitation, soit par les facteurs écologiques.

\section{Indice d'équitabilité de Pielou}

La comparaison des deux indices d'équitabilité de Pielou montre des variations entre l'AMP $(0,3967)$ et les zones non protégées à l'exploitation 
halieutique $(0,8653)$. Cette variation s'explique par la dominance de l'espèce E. melanopterus qui représente $49,9 \%$.

\section{Collecte de données primaires sur le terrain}

Les entretiens avec quelques acteurs à savoir le groupement des jeunes pêcheurs et le comité de gestion de l'AMP ont permis de ressortir :

37 espèces regroupées en 26 familles dans l'AMP ;

et 17 espèces regroupées dans 14 familles dans les ZNPEH.

Ces résultats sont similaires à ceux des inventaires qui ont montré 35 espèces avec 24 familles dans l'AMP et 18 espèces regroupées dans 15 familles en zones non protégées à l'exploitation halieutique.

La quasi-totalité des acteurs locaux sont conscients du rôle de l'AMP dans la préservation des ressources marines et côtières. Cependant certains pêcheurs trouvent des difficultés à reconnaitre les limites de l'AMP et de ses sous zones. Une baisse de la biodiversité marine est également constatée dans l'AMP et dans les zones non protégées à l'exploitation halieutique. Cela se justifie par les mauvaises pratiques de pêche et l'exploitation frauduleuse dans l'AMP. Pour les zones non protégées à l'exploitation halieutique, il faut ajouter également l'augmentation du nombre de pêcheur qui a pour conséquence la surpêche de la ressource ichtyologique. L'insuffisance de personnel dans l'AMP et au service de pêche mais également des moyens matériels et financiers constituent un réel problème pour la surveillance.

\section{Répartition spatiale des espèces}

L'AFC montre un peuplement où presque la totalité des espèces (23) soit $60,52 \%$ sont caractéristiques de l'intérieur de l'AMP. L'analyse comparée entre les deux zones a mis en évidence un nombre plus élevé d'espèces dans la zone protégée. La zone côtière de l'AMP de Joal-Fadiouth avec la forte diversité des habitats (mangroves, fond sableux, fond vaseux, estuaire) peut expliquer le développement important d'espèces. De même, la connectivité des deux zones et la mobilité des espèces favorisent le déplacement de certaines espèces. Ce qui fait qu'on trouve 09 espèces intermédiaires soit $23,68 \%$ et des espèces communes (15) soit 39,47\% entre les deux milieux.

Les deux espèces (E. melanopterus et $L$. dumerilii) se présentent comme les plus caractéristiques de la zone de l'AMP. Les espèces les plus communes dans les deux milieux sont Stephanolepis hispidus, Hemiramphus brasiliensis, Nicholsina collettei etc.

Concernant les niveaux trophiques de l'AMP, ils sont majoritairement dominés par les prédateurs de premier niveau (benthophage et généralistes) et les prédateurs de deuxième niveau (piscivores et généralistes). Cette situation est similaire à celle de l'AMP de Bamboung qui a montré également une forte dominance des prédateurs de premier et second niveaux (Sadio et al., 2015). 
Une situation qui peut s'expliquer par le fait que la plupart des juveniles des zooplanctonivores se rencontrent seulement dans les eaux peu profondes (moins de $9 \mathrm{~m}$ ), où ils trouvent un abri vis-à-vis des prédateurs. Ainsi on peut dire que l'AMP est peut-être une zone de nourricerie pour plusieurs espèces.

\section{Effet de réserve de l'Aire Marine Protégée}

Comparée aux ZNPEH, il ressort que l'ichtyofaune de l'AMP est plus diversifiée et elle est caractérisée par une richesse spécifique plus élevée. Même si, la diversité biologique dépendrait pour partie du niveau de l'exploitation des ressources, les résultats s'expliqueraient surtout par les conditions de vie pour les organismes à proximité des côtes (Rivierre, 2007). Les résultats entre les deux zones comparées montrent une augmentation significative en termes de diversité biologique et de richesse spécifique dans l'AMP.

L'augmentation de l'abondance des espèces dans l'AMP est un résultat très positif comparé aux ZNPEH. Ainsi, cette augmentation de l'abondance d'espèces peut s'expliquer par les mesures de gestion en vigueur au niveau de l'AMP qui sont favorables au développement de la diversité de faune ichtyologique. Par conséquent, toutes les espèces de faune ichtyologique recensées dans les zones de pêche non protégées se retrouvent dans l'AMP excepté 3 espèces (Chaetodipterus lippei, Scorpaena stephanica, Trachinocephalus myops).

\section{Conclusion}

L'étude a montré que la diversité ichtyologique est plus importante dans l'AMP de Joal-Fadiouth comparée aux pêcheries des zones non protégée à l'exploitation halieutique. La quasi-totalité des espèces de faune ichtyologique recensées dans les pêcheries des zones non protégées à l'exploitation halieutique se retrouvent dans l'AMP. Par conséquent, la stabilité du peuplement de poisson de l'AMP est liée aux mesures de gestions en vigueur au niveau qui sont favorables au développement de la diversité de faune ichtyologique. Les deux zones sont marquées par une forte dominance de prédateurs de premier niveau benthophage et de deuxième niveau généraliste qui occasionnent un phénomène de prédation accrue. L'étude comparative de la répartition spatiale des peuplements de poissons des deux sites d'étude a montré des modifications et des différences attribuables à l'effet réserve. Ce qui veut dire que les espèces ont plus une affinité à l'AMP qui par son statut de protection offre plus de bonnes conditions de vie. Du point de vue écologique, il y a une différence sur la composition des peuplements de poissons entre les deux sites. Aux vues des résultats, l'AMP apparait comme étant une réserve écologique dans cet écosystème marin et estuarien de la commune de Joal-Fadiouth. L'AMP de Joal-Fadiouth semble avoir un effet 
positif sur le peuplement en termes d'abondance mais est soumis également à une pêche frauduleuse.

\section{References:}

1. Albaret, J, J. (1999). Les peuplements des estuaires et des lagunes. In : Les Poissons Des Eaux Continentales Africaines : Diversité, Biologie, Écologie, Utilisation Par L'Homme (Eds. Lévêque C, Paugy D).IRD, Paris, 325-249.

2. ANSD (Agence Nationale de la Statistique et de la Démographie) (2011). Situation économique et sociale du Sénégal en 2010. 01p.

3. $B a, F$. (2017): Etude comparative de la diversité spécifique du peuplement ichtyofaunique, dans le réseau d'aires Marines Protégées entre 2015-2016 : CAS DE L'AMP DE CAYAR /Région de Thiès/ Sénégal. 5-6p

4. $B a, C$. (2000). Pêche. Atlas du Sénégal. Editions J.A. publ., Paris : 4041

5. Chabanne, D. (2007). Les Catégories bioécologiques des espèces de poissons des estuaires et lagunes de l'Afrique de l'Ouest. Mémoire de master, Université des Sciences et Techniques de Languedoc, 1-11p.

6. Diedhiou, D. K. (2015). Diversité des poissons de l'Aire Marine Protégée de Joal-Fadiouth. 12, 15 et 36p

7. Diouf, P.S. (1996). Les peuplements en poissons des milieux estuariens de l'Afrique de l'Ouest : l'exemple de l'estuaire hyperhalin du Sine-Saloum. Thèse Doct. Sci., Univ. Montpellier II, France : 267 $\mathrm{pp}$

8. Diallo, M.F. (2008). Situation de référence de la gouvernance des aires marines protégée de Joal-Fadiouth, Cacheu, Santa lucia, Branco et Rayo. Dakar : REPAO

9. Frontier, S. \& Pichod-Viale. (1991). Ecosystème : Structure, Fonctionnement, Evolution. Collection d'écologie. Masson, Paris, 392p.

10. Gray, J.S., Mclntyre, A.D. \& Štirn, J. (1992). Manuel des méthodes de recherche sur l'environnement aquatique. Onzième partie. Evaluation biologique de la pollution marine, eu égard en particulier au benthos. FAO Document technique sur les pêches, $\mathrm{N}^{\circ} 324,53$.

11. Gobat, J-M., Aragno, M. \&Matthey, W . (https://www.epflpress.org/auteur/1380/willy-matthey2010). Le sol vivant : bases de pédologie, biologie des sols. Presses Polytechniques et Universitaires Romandes, Lausanne, 569p.

12. Hansen, H.S. (2010). Modelling the future coastal zone urban development as implied by the IPCC SRES and assessing the impact from sea level rise. Landscape Urban Plan. 98 p. 
13. Hounsounou, L. \& Zacharie, S. (2014). Pêche à la senne de plage au Benin et durabilité des ressources halieutiques.

14. Lobry, J. (2004). Quel référentiel de fonctionnement pour les écosystèmes estuariens? Le cas des cortèges de poissons fréquentant l'estuaire de la Gironde. Thèse de doctorat, Université de Bordeaux I, Bordeaux.

15. Mbengue, B. (2016). Efficacité bioécologique des aires marines protégées : le cas de l'AMP de Joal-Fadiouth au Sénégal. Mémoire master, Université Nazi Boni, Burkina Faso, 56p.

16. Morris, D.W. (2006). Ecology: moving to the ideal free home. Nature, 443(7112), 645-646. doi:10.1038/443645a

17. Ndiaye, M. (2014). Biodiversité et structures bioécologiques de la faune ichtyologique de l'aire marine protégée de Cayar (Sénégal). 51 p.

18. Pauly, D. \& Froese, $R$. (2012). Commentaires sur l'état de la pêche et de l'aquaculture de la FAO, ou "SOFIA 2010". 36 p.

19. Roy, C. (1991). Les upwellings : le cadre physique des pêcheries côtières ouest-africaines. Pêcheries ouest-africaines. Cury P., Roy C. eds. ORSTOM publ, Paris : 38-66

20. Sarr S.M., Ndiaye M., Faye E. \& Diedhiou D.K. (2018). Richesse spécifique et abondance de la population ichtyologique de l'Aire Marine Protégée de l'Aire Marine Protégée de Joal- Fadiouth au Sénégal. International Journal of Innovation and Applied Studies ISSN 2028-9324 Vol. 24 No. 4 Nov. 2018, pp. 1664-1678. http://www.ijias.issr-journals.org/

21. Sarr S.M., Thiaw M. \& Mbengue B.A. (2018). Utilisation de paramètres bioécologiques pour analyser la biodiversité ichtyologique de l'Aire Marine Protégée de Joal-Fadiouth au Sénégal et sa périphérie non protégée à la pêche. European Scientific Journal November 2018 edition Vol.14, No.33 : 349-368, ISSN: 1857 - 7881 (Print) e - ISSN 1857- 7431. Doi: 10.19044/esj.2018.v14n33p349 URL:http://dx.doi.org/10.19044/esj.2018.v14n33p349

22. Sadio O., Simier, M., Ecoutin, J-M, Raffray, J., Laë, R. \& Tito de Morais, L. (2015). Effect of a marine protected area on tropical estuarine fish assemblages: Comparison between protected and unprotected sites in Senegal. Ocean et Coastal Management 116 257269.

23. Simier, M., Blanc L., Aliaume, C., Diouf P.S. \& Albaret J-J. (2004). Spatial and temporal structure of fish assemblages in an "inverse estuary", the Sine Saloum system (Senegal). Estuarine, Coastal and Shelf Science, 59(1), 69-86. En ligne http://www.dx.doi.org/10.1016/j.ecss.2003.08.002 
24. Thiam, N., Fall, M., Diadhiou, H.D., Diallo, A., Ndour, C., Sylla, M., Dème, L. \& Ndour, I. (2012). Réalisation des états de référence des zones de pêche protégées et suivi des récifs artificiels dans la zone du projet gestion durable des ressources halieutiques, Rapport final, Dakar : WWF-WAMPO. 